\title{
Primary Systemic Amyloidosis: a Case Report and Review of Clinical Features
}

\author{
Hu HQ, Wang SC, Cao X and Cao BZ \\ Department of Neurology, Jinan General Hospital of Chinese People's Liberation Army, Jinan, China
}

\begin{abstract}
:
Primary systemic amyloidosis is a rare disorder that has multisystemic manifestations. The diagnosis is very difficult because of non-specific clinical signs. We report a patient with primary systemic amyloidosis manifesting as peripheral neuropathy, nephrotic syndrome, gastrointestinal syndrome, orthostatic hypotension, pericardial fluid, weight loss and so on. Histopathological examination of kidney and sural nerve showed Congo red positive deposits. Primary systemic amyloidosis should be considered in any patient older than 40 years who has the following symptoms and signs: fatigue, weight loss, edema, paresthesias, muscle weakness, noninfectious diarrhea, skin and soft-tissue lesions, and the complications of nephrotic syndrome, congestive heart failure (not on an ischemic basis), peripheral neuropathy, or unexplained hepatomegaly. All patients need the diagnosis confirmed histologically by biopsy of an involved organ.
\end{abstract}

\section{KEYWORDS: Primary systemic amyloidosis, amyloidosis, clinical features}

\section{CASE REPORT}

A 66-year-old female presented with bilateral lower limb asthenia and feet ache with abdominal discomfort four years ago, bilateral upper limb and hands asthenia, two years ago, which progressively deteriorated with general weakness one year ago. Her body weight reduced about $28 \mathrm{~kg}$ and the blood pressure was unstable and usually low since the disease. The blood pressure was about $120 / 80 \mathrm{mmHg}$ before the disease and was about $100 / 60 \mathrm{mmHg}$ after the disease. Her past history, personal history, family history were unremarkable.

Physical examination revealed $\mathrm{T}-36.8^{\circ} \mathrm{C}, \mathrm{P}-76 \mathrm{bpm}$, RR-20 bpm, BP 130/90 mmHg. Her development was normal, and she represented with thin body, poor nutrition, clear mind. Skin was without erythema, papula and ulcer. Heart, lung and abdomen were normal. Both lower extremities showed moderate edema. Normal mini-mental state examination, clear words, normal encephalic nerves, emaciated four limbs. Obvious atrophy of interosseous muscles of hands, muscles of thenar and hypothenar eminences, both tibial muscles, gastrocnemius muscles. Muscle power, proximal both upper extremities $\mathrm{V}$, distal end III, obviously restrict adduction and abduction of both fingers; both lower extremities proximal end IV, distal

Corresponding author

Hu Huai-qiang

Jinan General Hospital of Chinese People's

Liberation Army, Jinan, China

e-mail: huhuaiqiang@126.com end III, bilateral feet drop. Sensory examination, the algaesthesis and vibratory sense were impaired below bilateral elbow-joints, especially the both wrist joints; the algaesthesis, tactile impression, joint position and vibratory sense were lost below the knee joints. Limb tendinous reflexes, Kernig's sign and Brudzinski's signs were negative. Urination and defecation without incontinence.

Blood cell analysis: white blood cell count $5.27 \times 10^{9} / \mathrm{L}$, red blood cell count $3.42 \times 10^{12} / \mathrm{L}$, hemoglobin $104 \mathrm{~g} / \mathrm{L}$; liver function: total protein $58.9 \mathrm{~g} / \mathrm{L}$, albumin: $24.8 \mathrm{~g} / \mathrm{L}$, globulin $34.1 \mathrm{~g} / \mathrm{L}, \mathrm{A} / \mathrm{G} 0.73$; erythrocyte sedimentation rate $34 \mathrm{~mm} / \mathrm{h}$; immunoglobulin $\mathrm{G} 17.9 \mathrm{~g} / \mathrm{L}$; complement C3 $0.779 \mathrm{~g} / \mathrm{L}$; saccharide antigen CA125 $185.2 \mathrm{U} / \mathrm{ml}$; urine analysis: protein $(2+)$, occult blood $(+)$. Blood electrolytes, cholesterol, coagulation indicator, immunoglobulin A, immunoglobulin $M$, immunoglobulin light chain protein, complement $\mathrm{C} 4$, antistreptolycin $\mathrm{O}$, rheumatoid factor, $\mathrm{C}$ reactive protein, thyroid function(FT3,FT4,TSH), CA19-9, carcino-embryonic antigen and stool routine were all normal. Hepatitis B virus surface antigen, hepatitis $C$ virus antibody, human immunodeficiency virus antibody, treponema pallidum antibody, rheumatism polypeptide antibody(anti-SM, anti-U1RNP, anti-SSA, anti-SSB, anti-SCL-70, anti-JO-1, anti-HVP, anti-Liu, anti-RA-54), total antinuclear antibodies(antinuclear antibody, Ds-DNA, Sm/RNP, SSA/SSB) were all negative.

Electrocardiogram indicated sinus rhythm, low voltage in the limb leads and low $T$ waves. Chest $X$ ray showed left pachynsis synechial pleurae and aortosclerosis. Abdominal organs were examined by the color b-sonography. The liver was normal. The right kidney was $102 \times 45 \mathrm{~mm}$, left kidney was $110 \times 52 \mathrm{~mm}$, normal morphous, fuzzy demarcation between cor- 
tex and medulla, cortical thinning, normal aggregation system. The chronic superficial gastritis, gastric retention, proctitis and colonic diverticula were diagnosed by the digestive endoscopy. The results of mucous membrane of rectum biopsy were CD68 (+), CK (-), S-100 (-), CD1a (-), PAS (+), acid-fast stain (-), Congo red stain $(-)$, which showed chronic inflammation. The pelvis and skull were normal by computerized tomography. The bone marrow biopsy showed the active hyperplasia and normal plasma cell.

The echocardiogram found increased interventricular septum and left ventricular wall thickness with normal echo; columna papillares were thickened; mitral, tricuspid and aortic valves showed mild regurgitation; with moderate pericardial effusion, mild pulmonary hypertension, abnormal left ventricular filling existed. By total body opacification of positron emission computed tomography, both lower lung (especially the left lower lung) showed pulmonary consolidation; the density of both thyroids were uneven; there was myocardial hypertrophy with pericardial effusion; the density of subcutaneous fat all over the body was raised; the metabolism of fluorodeoxyglucose raised in the above-mentioned focus areas and distal end of rectum. To further clarify the disease, the sural nerve and renal biopsy were obtained (Figures 1 4).

\section{DISCUSSION}

Primary systemic amyloidosis is a rare systemic disorder of protein metabolism with progressive extracellular deposition of insoluble fibrillary protein, disorganization of tissue architecture, and subsequent organ dysfunction. The incidence rate is reported as $0.09 \%$ 0.80\%, ${ }^{1}$ the onset age between 40 and 60; and males are more than females in the primary systemic amyloidosis. Clinical manifestations are complex, variable and lack specificity, so early diagnosis is very difficult in this disorder.

The outstanding clinical manifestations were peripheral lesion, gastrointestinal symptom and renal insufficiency. The case presented with the loss of algaesthesis, tactile sense, joint position sense, vibratory sense that gradually spread from the end of both lower limbs to both hands and upper limbs, and involved the whole body. The sensory symptoms were more serious than cinesipathy, and the lower limbs were worse than the upper limbs. The EMG of the patient revealed multiple neuraxon and myelin processes of aesthema and movement and damaged various diameter nerve fibers. In addition, the esthesionosis, asymmetrical multiple single radiculoneuropathy, demyelinate radiculoneuropathy and peripheral neuropathy combined with motor neuron disease are rare in the atypical cases. ${ }^{2}$

The gastrointestinal symptoms were also prominent, which manifested as periumbilical discomfort, diarrhea, constipation and anorexia. Chronic superficial gastritis was diagnosed by multiple gastroscopies, but treated without obvious effect. In addition, liver disfunction was present and weight loss was obvious.
Weight loss is most common signs in all patients, with a median weight loss of 30 pounds (range, 2 to 134 pounds).$^{3}$

Approximately $50 \%$ of patients had renal insufficiency at the time of diagnosis. Proteinuria was found in more than $90 \% .{ }^{4}$ Edema and albuminuria are the most common clinical manifestations in the case, but hematuria is rare. About $50 \%$ of patients experience some cardiac manifestation related to their disease, and at least $25 \%$ have congestive heart failure. ${ }^{5}$ Although the patient had no cardiac symptoms the electrocardiogram and echocardiogram found cardiomyopathy and pericardial effusion. The echocardiogram revealed myocardial hypertrophy and normal ventricular cavity size, but the electrocardiogram reveals low voltage in the limb leads. So, cardiac amyloidosis should be suspected in any patient who presents with restrictive cardiomyopathy, prominent signs of right-sided heart failure, or left sided heart failure in the absence of ischemic disease- particularly in patients with known systemic amyloidosis. ${ }^{6}$ The echocardiogram and electrocardiography contribute to early diagnosis of amyloid heart disease.

In conclusion, substantial numbers of patients with primary systemic amyloidosis have peripheral neuropathy, nephrotic syndrome, congestive heart failure, sprue, or orthostatic hypotension. The patient appears with the following unexplained symptoms and signs: fatigue, weight loss, edema, dyspnea, paresthesias, muscle weakness, hepatomegaly, renal failure with normal renal size, myocardial hypertrophy with low voltage, dysfunction of liver without hepatitis, damaged peripheral nervous system and noninfectious diarrhea. Pathological diagnosis today is still based largely on Congo red staining of the amyloid fibril subunit when the typical apple-green birefringence is seen under polarised light. At present, positron emission computed tomography contributes to diagnoses of primary systemic amyloidosis.

\section{REFERENCES}

1 Black RM. Amyloidosis and light-chain deposition disease. In: Black RM, ed . Clinical problems in nephrology [M]. Boston: Little, Brown, 1996. 305-314.

2 Vucic S, Chong PST, Cros D. Atypical presentations of primary amyloid neuropathy. Muscle Nerve 2003; 28:696-702

Hayman SR, Lacy MQ, Kyle RA, et al. Primary systemic amyloidosis: a cause of malabsorption syndrome. Am J Med 2001; 111(7):53540

4 Kyle RA, Bayrd ED. Amyloidosis: review of 236 cases. Medicine 1975; 54(4):271-99

5 Abraham RS, Geyer SM, Price-Troska TL, et al. Immunoglobulin light chain variable (V) 
region genes influence clinical presentation and outcome in light chain-associated amyloi dosis (AL). Blood 2003; 101:3801-8

Gertz MA, Lacy MQ, Dispenzieri A. Amyloidosis; recognition, confirmation, prognosis, and therapy. Mayo Clin Proc 1999; 74; 490-4

Figure 1. The part blood vessel walls of renal interstitium were stained by Congo red; some of amyloid are indicated by arrows (original magnification $\times 400$ ).

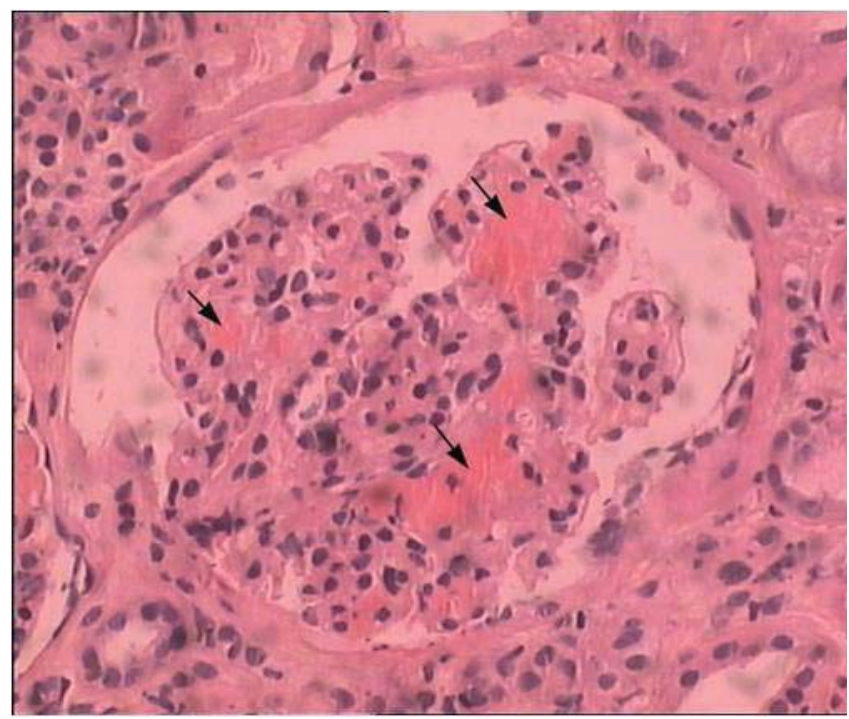

Figure 2. Some of amyloid are indicated by arrows in the nervus suralis biopsy (Congo red staining original magnification $\times 400$ ).

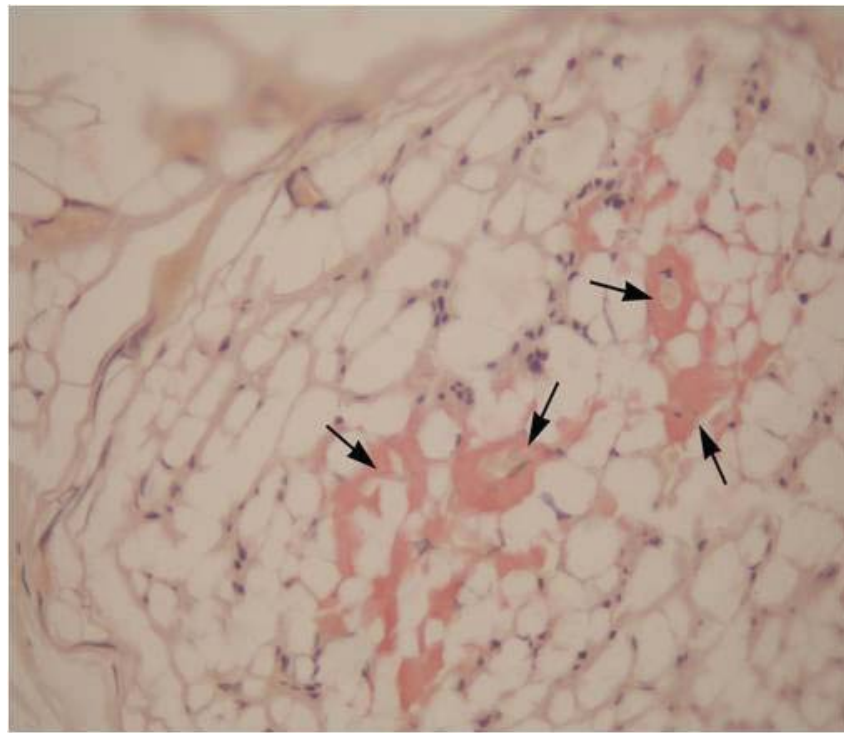

Figure 3 and 4. Myelin disappearing, peripheral nerve structural damage like honeycomb appearance (Figure 3: toluidine blue staining original magnification $\times 400$, Figure 4: HE staining original magnification $\times 400$ ).
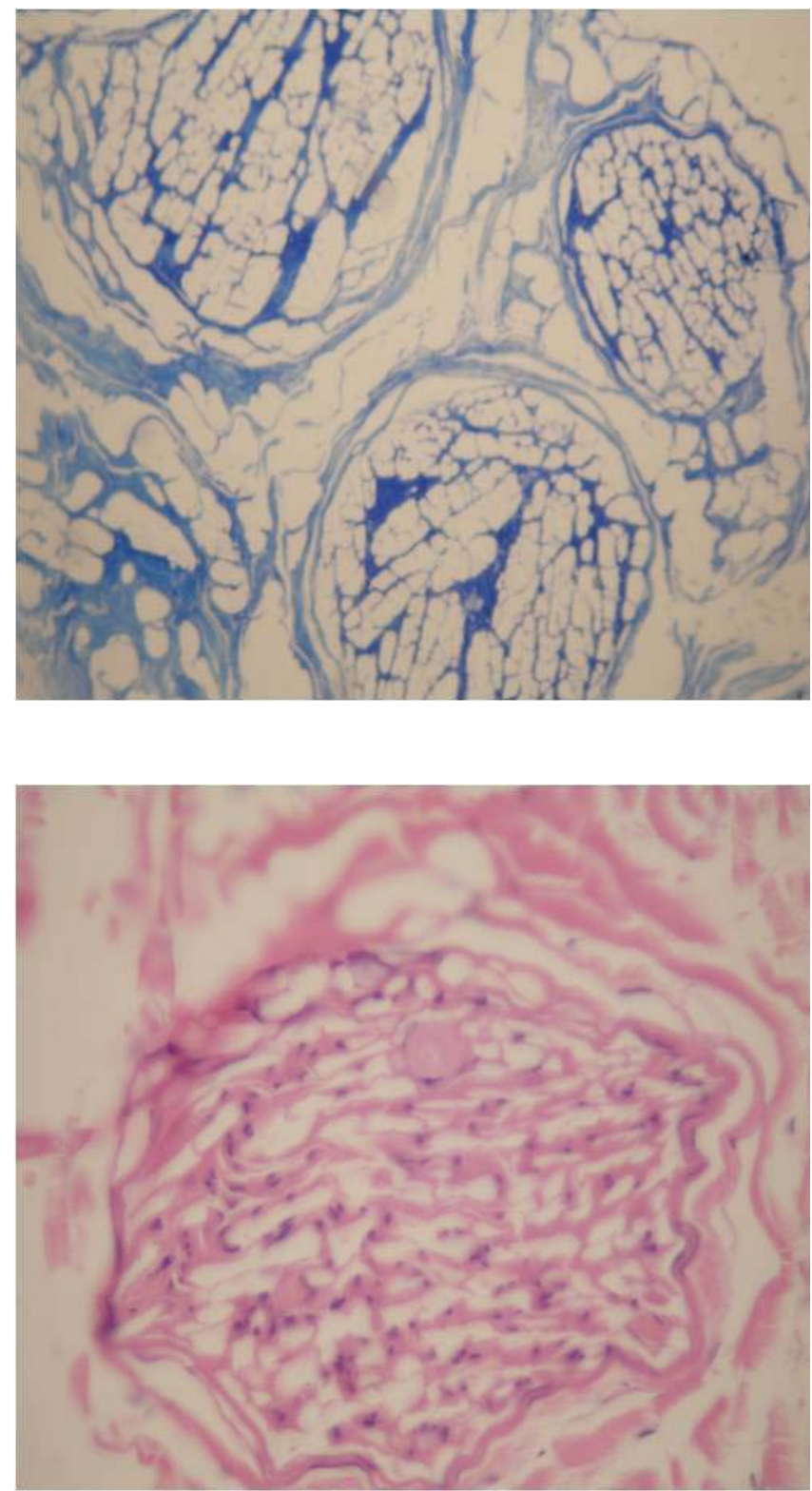
